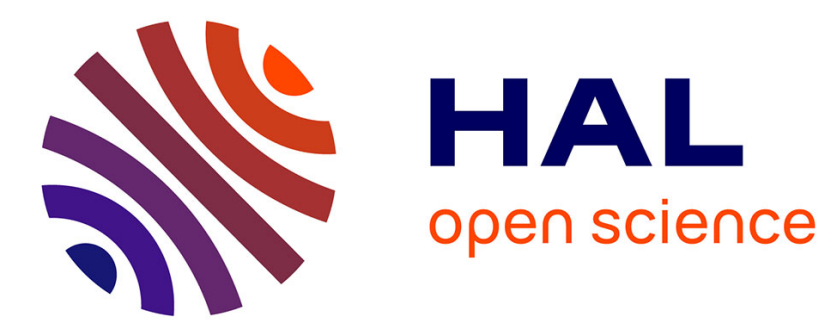

\title{
Wall effects on the transportation of a cylindrical particle in power-law fluids
}

\author{
Antoine Despeyroux, Abdelhak Ambari, Abderrahim Ben Richou
}

\section{To cite this version:}

Antoine Despeyroux, Abdelhak Ambari, Abderrahim Ben Richou. Wall effects on the transportation of a cylindrical particle in power-law fluids. Journal of Non-Newtonian Fluid Mechanics, 2011, 166 (19-20), pp.1173-1182. 10.1016/j.jnnfm.2011.07.004 . hal-01060920

\section{HAL Id: hal-01060920 \\ https://hal.science/hal-01060920}

Submitted on 4 Sep 2014

HAL is a multi-disciplinary open access archive for the deposit and dissemination of scientific research documents, whether they are published or not. The documents may come from teaching and research institutions in France or abroad, or from public or private research centers.
L'archive ouverte pluridisciplinaire HAL, est destinée au dépôt et à la diffusion de documents scientifiques de niveau recherche, publiés ou non, émanant des établissements d'enseignement et de recherche français ou étrangers, des laboratoires publics ou privés. 


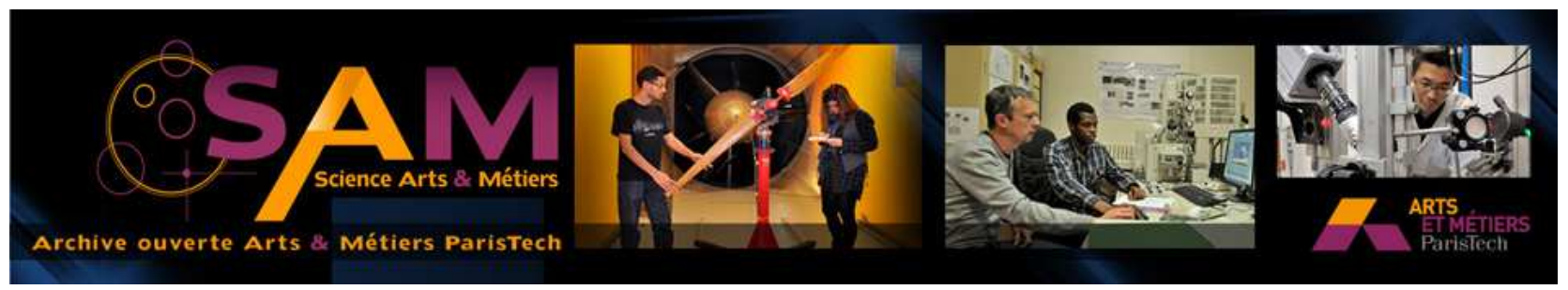

Science Arts \& Métiers (SAM)

is an open access repository that collects the work of Arts et Métiers ParisTech researchers and makes it freely available over the web where possible.

This is an author-deposited version published in: http://sam.ensam.eu

Handle ID: .http://hdl.handle.net/10985/8454

\section{To cite this version :}

Antoine DESPEYROUX, Abdelhak AMBARI, Abderrahim BEN RICHOU - Wall effects on the transportation of a cylindrical particle in power-law fluids - Journal of Non-Newtonian Fluid Mechanics - Vol. 166, n¹9-20, p.1173-1182 - 2011 


\title{
Wall effects on the transportation of a cylindrical particle in power-law fluids
}

\author{
A. Despeyroux, A. Ambari* , A. Ben Richou \\ Arts et Métiers ParisTech, 2 bd du Ronceray, Angers 49035, France
}

\begin{abstract}
A B S T R A C T
The present work deals with the numerical calculation of the Stokes-type drag undergone by a cylindrical particle perpendicularly to its axis in a power-law fluid. In unbounded medium, as all data are not available yet, we provide a numerical solution for the pseudoplastic fluid. Indeed, the Stokes-type solution exists because the Stokes' paradox does not take place anymore. We show a high sensitivity of the solution to the confinement, and the appearance of the inertia in the proximity of the Newtonian case, where the Stokes' paradox takes place. For unbounded medium, avoiding these traps, we show that the drag is zero for Newtonian and dilatant fluids. But in the bounded one, the Stokes-type regime is recovered for Newtonian and dilatant fluids. We give also a physical explanation of this effect which is due to the reduction of the hydrodynamic screen length, for pseudoplastic fluids. Once the solution of the unbounded medium has been obtained, we give a solution for the confined medium numerically and asymptotically. We also highlight the consequence of the confinement and the backflow on the settling velocity of a fiber perpendicularly to its axis in a slit. Using the dynamic mesh technique, we give the actual transportation velocity in a power-law "Poiseuille flow", versus the confinement parameter and the fluidity index, induced by the hydrodynamic interactions.
\end{abstract}

Keywords:

Power-law fluid

Cylinder

Stokes-type force

Hydrodynamic interactions

Non-Newtonian fluids

Stokes' paradox

\section{Introduction}

Mechanical properties of plastics are enhanced by the addition of fibers which can take different aspects. In particular, short fibers filled thermoplastics are often employed in the injection of composite materials processes. The mechanical properties of the composite parts obtained depend on the kinematics of the process flow. In turn, this kinematics depends on the rheological properties of the suspension, which itself relies on the dynamics of each particle. This dynamics has to take into account of their hydrodynamic interactions with the wall of the container and with other particles. Indeed, these effects are often neglected in modeling despite the high concentrations used in industrial processes. This commonly used hypothesis (which is true only in dilute regime) leads to the wrong estimation of the real velocity of transportation and rotation of the particle, particularly in concentrated regime. Let us also recall that these interactions control the orientation distribution function of fibers, which is not discussed here. In fact, the effect of these interactions has been tackled by many researchers, as Sepehr et al. [1]. But in this study, due to the complexity of the problem, we tried to give a physical understanding of the effect of these interactions in a power-law fluid, which can induce a more or less high range of the screen length velocity field, controlled through the fluidity index. Then, the first step to understand these

\footnotetext{
* Corresponding author. Tel.: +33 241207362; fax: +33 241207362.

E-mail addresses: ambari@ensam.eu, abdelhak.ambari@angers.ensam.fr (A. Ambari).
}

interactions leads us to study the basic hydrodynamics related to the transportation of an individual circular cylindrical particle in a confined situation. The justification of the use of this model in Newtonian fluids at high concentration has been made by Champmartin and Ambari [2]. To do so, we have chosen a simple model constituted by an infinitely long circular cylinder of radius $a$, translating uniformly midway perpendicularly to its axis, between two parallel plane walls distant of $2 b$ (Fig. 1 ). It is important to recall that some viscoelastic fluids are able to act on the structure formation in suspensions $[3,4]$. In the present study, as the elongational strain rates upstream and downstream of the particle are low enough (low Deborah number), we are concerned in a first step only by the effect of the shear thinning or shear thickening behavior on these hydrodynamic interactions, in a non-Newtonian suspending fluid. In this case, the rheology of the suspending fluid is approximated by the most commonly used Ostwald-de Waele law.

First of all, in an unlimited medium, there is no solution for the Stokes equation for a cylinder in Newtonian fluid [5-7]. The consequence of this paradox is that the drag per unit length of the cylinder tends towards zero when $R e \rightarrow 0$ as confirmed by Lamb's solution [7]. $F_{x}^{\text {Lamb }}=4 \pi \mu U_{x} /[1 / 2-\gamma+\ln (8 / \operatorname{Re})]$ with $\gamma=0.5772 \ldots$ the Euler constant. Nevertheless, we showed in Newtonian fluid that the Stokes-type solution is obtained in the confined medium at low Reynolds numbers. Otherwise, the Stokes-type solution is also obtained for a finite length rod in the approximation of the slender body theory given by Batchelor [8]. Besides, when the suspending fluid exhibits a shear thinning or shear thickening nonNewtonian rheology, the hydrodynamic screen length is strongly 


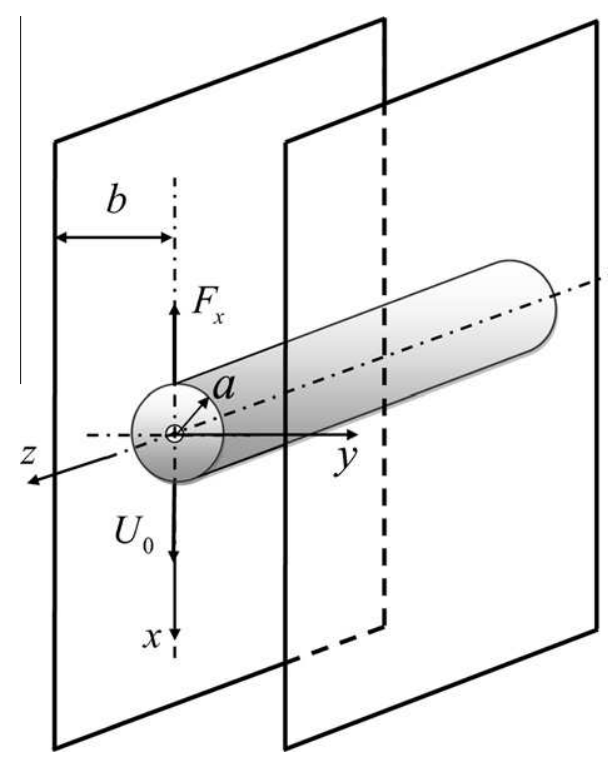

Fig. 1. Sketch of the motion of a cylinder perpendicularly to its axis in the midplane position between two parallel walls.

affected. Then, as we will see, in the shear thinning case, the localization of the flow near the cylinder leads to expect the disappearance of the Stokes' paradox for $n<1$ as the confinement does. However, in the Newtonian and shear thickening fluids, the increase of the long range hydrodynamic perturbation around a cylinder should imply the existence of Stokes' paradox. The first person expecting this behavior was Tanner [9] followed by Marušić-Paloka [10].

Finally, in this study, we are dealing with the evaluation of the drag undergone by a cylindrical particle, transported through a power-law fluid in confined media at low Reynolds numbers. Then, we provide an accurate calculation of the effect of the hydrodynamic interactions numerically and asymptotically.

Taking into account of all these results, and particularly the sensitivity to the inertia, we will give a study on the drag submitted by a cylinder in a confined situation and we will calculate the relative velocity of the transportation of a free cylindrical particle in a power-law "Poiseuille flow".

\section{Mathematical formulation and numerical approach}

\subsection{Mathematical formulation}

The problem we are dealing with is the flow of a fluid around a cylinder, of radius $a$, falling midway and parallel to two planes distant of $2 b$, shown schematically in Fig. 1 . For easiness of the calculations, it is assumed that the cylinder is set and both walls are moving with the fluid velocity $-U_{0} \boldsymbol{e}_{x}$. In fact, as the apparent viscosity of the power-law fluid does not depend on the sign of the velocity gradient, both procedures are equivalent. The flow is governed by the usual conservation equations for mass and momentum under isothermal conditions, i.e.

$$
\left\{\begin{array}{l}
\nabla \cdot \boldsymbol{V}=0 \\
\rho\left(\partial_{t} \boldsymbol{V}+(\boldsymbol{V} \cdot \nabla) \boldsymbol{V}\right)=-\nabla p+\nabla \cdot \underline{\underline{\tau}}
\end{array}\right.
$$

where $\rho$ is the fluid density, $p$ the pressure and $\tau$ the extra-stress tensor for the power-law fluid. At very low generalized Reynolds numbers $R e_{n}=\rho U_{0}^{2-n}(2 a)^{n} / m$, the governing equations are written in a dimensionless form using the following dimensionless variables: $x=x^{*} / a, y=y^{*} / a, \boldsymbol{V}=\boldsymbol{V}^{*} / U_{0}, t=t^{*} / \tau_{v}, \quad p=a p^{*} / \mu_{a p 0} U_{0}, \mu_{a p}$ $=\mu_{a p}^{*} / \mu_{a p 0}, \psi=\psi^{*} / a^{2} U_{0}$ and $\omega=\omega^{*} U_{0} / a$ where $\mu_{a p 0}=m\left(U_{0} / a\right)^{n-1}$ and $\tau_{v}=\rho a^{2} / \mu_{a p 0}$ is the characteristic time of vorticity diffusion and the superscript $(*)$ represents the dimensional quantities. Under these conditions the problem is controlled by the three following parameters: the aspect ratio $k=a / b$ which is the geometrical confinement parameter, the power-law fluidity index $n$ and the generalized Reynolds number $R e_{n}$. At low Reynolds numbers, these equations can be expressed in the stream function $\psi$ and vorticity $\omega$ formulation as:

$$
\begin{aligned}
\frac{\partial \omega}{\partial t}+\frac{R e_{n}}{2^{n}}\left(u \frac{\partial \omega}{\partial x}+v \frac{\partial \omega}{\partial y}\right)= & \mu_{a p}\left[\frac{\partial^{2} \omega}{\partial x^{2}}+\frac{\partial^{2} \omega}{\partial y^{2}}\right]+2 \frac{\partial \mu_{a p}}{\partial x} \frac{\partial \omega}{\partial x} \\
& +2 \frac{\partial \mu_{a p}}{\partial y} \frac{\partial \omega}{\partial y}+S
\end{aligned}
$$

$-\omega=\frac{\partial^{2} \psi}{\partial x^{2}}+\frac{\partial^{2} \psi}{\partial y^{2}}$

where,

$$
\begin{aligned}
& \left.S=2 \frac{\partial^{2} \mu_{a p}}{\partial x \partial y}\left(\frac{\partial v}{\partial y}-\frac{\partial u}{\partial x}\right)+\left(\frac{\partial u}{\partial y}+\frac{\partial v}{\partial x}\right) \frac{\partial^{2} \mu_{a p}}{\partial x^{2}}-\frac{\partial^{2} \mu_{a p}}{\partial y^{2}}\right) \\
& \mu_{a p}=\left[2\left(\frac{\partial u}{\partial x}\right)^{2}+2\left(\frac{\partial v}{\partial y}\right)^{2}+\left(\frac{\partial u}{\partial y}+\frac{\partial v}{\partial x}\right)^{2}\right]^{(n-1) / 2} \\
& u=\frac{\partial \psi}{\partial y}, \quad v=-\frac{\partial \psi}{\partial x}, \quad \text { and } \quad \omega=\left(\frac{\partial v}{\partial x}-\frac{\partial u}{\partial y}\right)
\end{aligned}
$$

The velocity boundary conditions become:

(i) on the plane walls: $\boldsymbol{V}(x, y= \pm 1 / k, t)=-\boldsymbol{e}_{x}$. Thus $\psi(x, y$ $=b / a)=b / a$;

(ii) on the cylinder, the no-slip boundary condition $\mathbf{V}=\mathbf{0}$ on the fixed cylinder conducts to $\psi(x, y)=$ Cst for all points $(x, y)$ of the circular profile;

(iii) upstream and downstream, the flow is uniform of velocity $\boldsymbol{V}(x, y \rightarrow \pm \infty, t)=-\boldsymbol{e}_{x} . \quad$ Thus $\quad \psi(x \rightarrow \pm \infty, y)=y \quad$ and $\omega(x \rightarrow \pm \infty, y)=0$.

\subsection{Numerical method}

To compute solutions for $\psi$ and $\omega$ corresponding to the flow around a cylindrical particle, the governing Eqs. (1) and (2) are rewritten in a curvilinear and orthogonal frame $(X, Y)$ matching perfectly the boundaries of the cylinder and the walls [11]. Using the singularities method we generate a conformal mapping from curved borders to a rectangular one. This grid is built with the equipotential lines $X(x, y)=$ Cst and streamlines $Y(x, y)=$ Cst, corresponding to the flow of an inviscid fluid (see Fig. 2, given here for the confinements $k=0.99$ and $k=0.44$ ) [11]. The meshing is performed using a combination of the singularities method and the finite differences technique.

The computation of the stream and vorticity functions equations is carried out by the finite differences method using the successive over-relaxation (SOR) and the alternating direction implicit (ADI) techniques [12-16] respectively to calculate the functions $\psi$ and $\omega$ of the fluid. For a given confinement parameter $k$, once the stream function and the vorticity have been obtained, the dimensionless wall correction factor of the drag $\lambda(n, k)$ exerted by the fluid on the particle, is obtained by the normalization of the actual force by the characteristic one $F_{0}=4 \pi m\left(U_{0} / 2 a\right)^{n-1} U_{0}$ (Eq. (3)). The total actual drag is computed by integrating the viscous and pressure stresses over the surface of the cylinder:

$\lambda(n, k)=F(n, k) / F_{0}$

For the computation, we used a sixteen parallelized core cluster and we successfully checked the influence of the spatial resolution for 


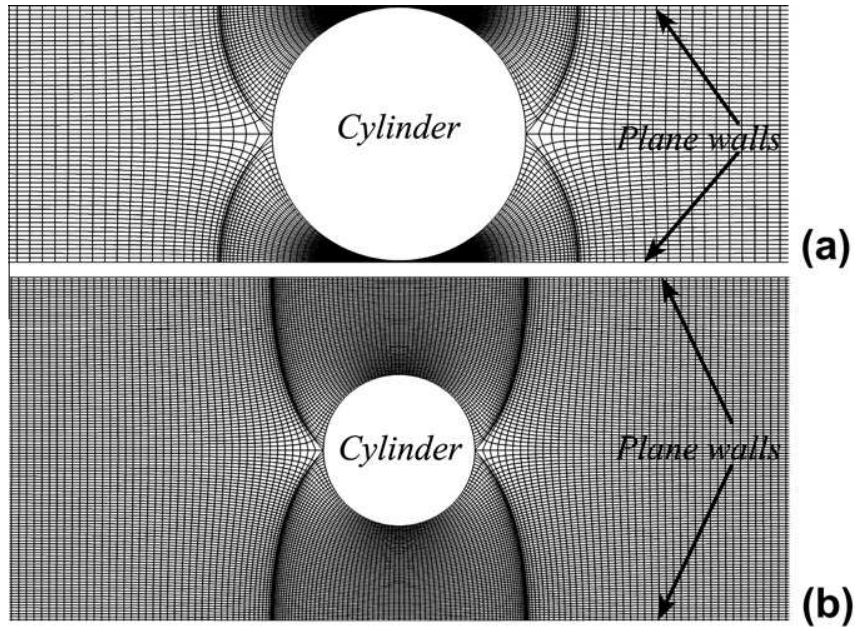

Fig. 2. The orthogonal grid structure for different aspect ratios: (a) $k=0.99$, (b) $k=0.44$ corresponding to the equipotential and the streamlines.

each confinement. The convergence of the computation is supposed to be reached when the following criterion is checked:

$\left|1-\lambda^{l}(n, k) / \lambda^{l+1}(n, k)\right|<10^{-6}$

\subsection{Alternative numerical method}

To validate this numerical method, we verified the numerical results by the use of the finite volume CFD code FLUENT, where the SIMPLE algorithm was employed with a second order scheme on a structured mesh. The convergence criteria used is the same as the one used previously. All the data obtained by this CFD code corroborate our numerical technique. Thus, the accuracy of the two numerical methods is mutually confirmed.

\section{Stokes-type force in unbounded medium}

In this problem, we give first the results concerning the unbounded medium and why the problem is sensitive to the inertia when Stokes' paradox would take place. So, when the "Stokes-type solution" exists, it is not difficult to establish, using dimensional analysis, that the correction factor $\lambda(n, k)$ of the force undergone by the cylinder translating at a constant velocity in the midplane (Fig. 1) is given by:

$\boldsymbol{F}(n, k) / l=-4 \pi m\left(\frac{U_{0}}{2 a}\right)^{n-1} U_{0} \lambda(n, k) \boldsymbol{e}_{x}$

where $k=a / b$ is the confinement factor, $m$ and $n$ are respectively the consistency coefficient ( $\mathrm{Pa} s^{n}$ ) and the power-law index of the fluid corresponding to the following constitutive equation:

$\sigma_{i j}=-p \delta_{i j}+2 m\left|2 d_{k l} d_{k l}\right|^{(n-1) / 2} d_{i j}$

where $\sigma_{i j}$ are the Cauchy stress tensor components, $d_{i j}=\frac{1}{2}\left(V_{i, j}+V_{j, i}\right)$ are the rate of deformation tensor components and $p$ the pressure. To normalize the results, we calculated numerically, in unbounded medium, the correction factor $\lambda(n, k=0)$ using the numerical method presented in Sections 2.1 and 2.2. In fact, the correction factor for the drag in unbounded medium for a cylindrical particle $\lambda(n, k=0)$ has not received as much attention as in the case of a spherical particle. In the Fig. 3, we compared the numerical results to those obtained first by Tanner [9] (who used a perturbation method). His first results, for $k=0$, are in agreement with the present numerical results only for $0.7 \leqslant n<1$. But his second results, obtained by the

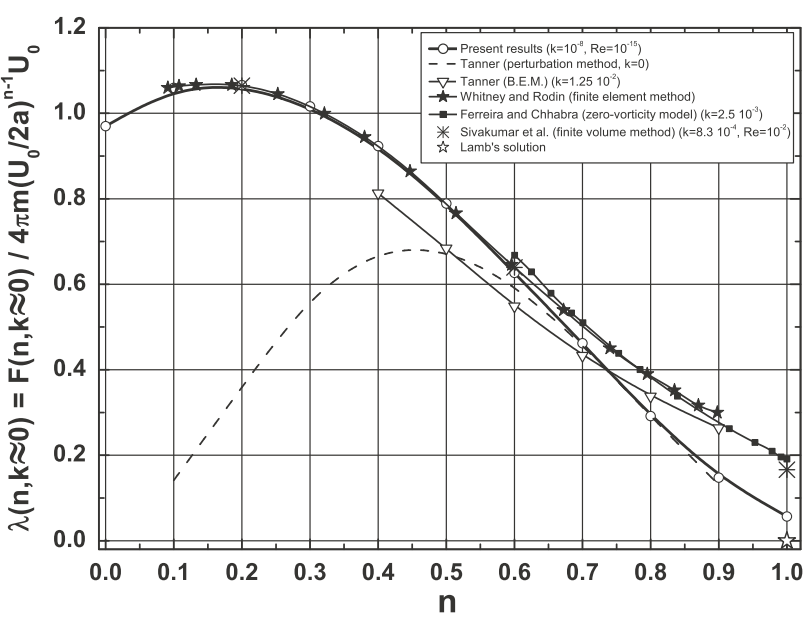

Fig. 3. Comparison between the present numerical results and those obtained previously by different authors.

boundary element method for $k \approx 10^{-2}$, are not in accordance with the present results. Ferreira and Chhabra $\left(k=2.510^{-3}\right)$ [17], who give values for $0.6 \leqslant n<1$, overestimate the calculation of $\lambda(n, k=0)$, probably due to the effect of the confinement, as we will show in this section. Whitney and Rodin [18], using finite element method, also overestimate the calculation of this correction factor for $0.6 \leqslant n \leqslant 0.9$, but give numerical results in good accordance with the present ones, for $0.1 \leqslant n<0.6$, as shown in this same figure. Similar results have been obtained by Sivakumar et al. [19] and are added on the Fig. 3. Faced to this situation, we try to give an accurate numerical result, taking into account of the difficulty related to the influence of the confinement and the inertia which appears earlier at low generalized Reynolds numbers for $n \approx 1$. In fact, for low values of $n(n=0.6)$, we show in the Fig. 4 a that the inertia appears at relatively high Reynolds numbers (at $R e_{n}=10^{-1}$, we deviate from the plateau regime), and the low confinement needed to achieve the unbounded medium is at least about $10^{-3}$. Indeed, due to the inhomogeneity of the viscosity, the non-inertial regime takes place while we do not leave the plateau regime. Contrary to the previous case, for $n=0.9$, which is in the vicinity of the Newtonian case, the Fig. 4b shows that the inertia takes place above $R e_{n}=10^{-6}$. This fact is due to the increase of the sensitivity to the inertia in the proximity of the critical point where the Stokes' paradox takes place. Faced to both results, it is easy to understand why low values of the confinement and the Reynolds number are needed to obtain an unbounded medium, as shown in the Fig. 5. Thus, the overestimation of the correction factor may be due either to the higher confinement or the higher generalized Reynolds number. This same figure shows also that the drag force is not zero for $n \geqslant 1$ for relatively high confinements, in contrary to the expected results in this range of $n$ where the Stokes' paradox takes place and the drag force must be zero. This result shows that the Stokes type force exists in this last range in a bounded medium, as in the Newtonian case. The proof of this result is given in the Fig. 4c, showing that the plateau regime can be achieved. Moreover, for information only, in the Fig. 5 , we also give the values of $\lambda(n, k=0)$ obtained by the classical minimum of dissipation method which was applied by Tomita [20] and Wasserman and Slattery [21], where the approximate stream function $\psi$ used by Tanner [9] for its perturbation calculation, depending on two parameters $(p, q)$, is employed:

$\psi=U_{0} r \sin \theta\left[1+\frac{q}{p-q}\left(\frac{r}{a}\right)^{p}+\frac{p}{p-q}\left(\frac{r}{a}\right)^{q}\right]$

The approximate solution for the force in an unbounded medium was obtained by the minimization of the energy dissipation. The 

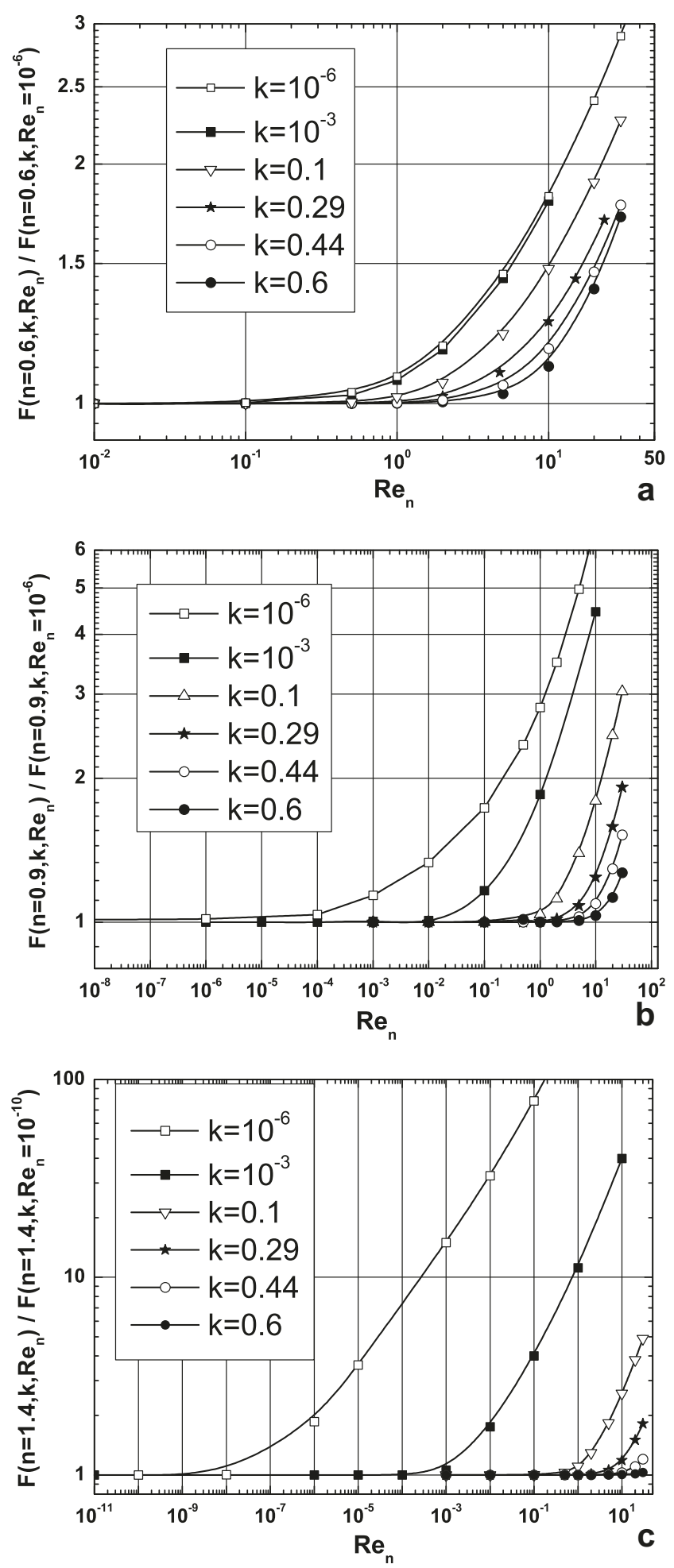

Fig. 4. Influence of the confinement on the critical Reynolds number marking the rise of the inertia: (a) for $n=0.6$, (b) for $n=0.9$, (c) for $n=1.4$.

correction factor for the $\operatorname{drag} F(n, k=0)$ is then given by the following expression:

$\lambda(n, k=0)=\frac{(2 a)^{n-1}}{2 \pi m U_{0}^{n+1}} \int_{a}^{\infty} \int_{0}^{\pi} \phi r \mathrm{~d} \theta \mathrm{d} r$

where:

$\phi=m\left[4\left(d_{r r}^{2}+d_{r \theta}^{2}\right)\right]^{(n+1) / 2}$

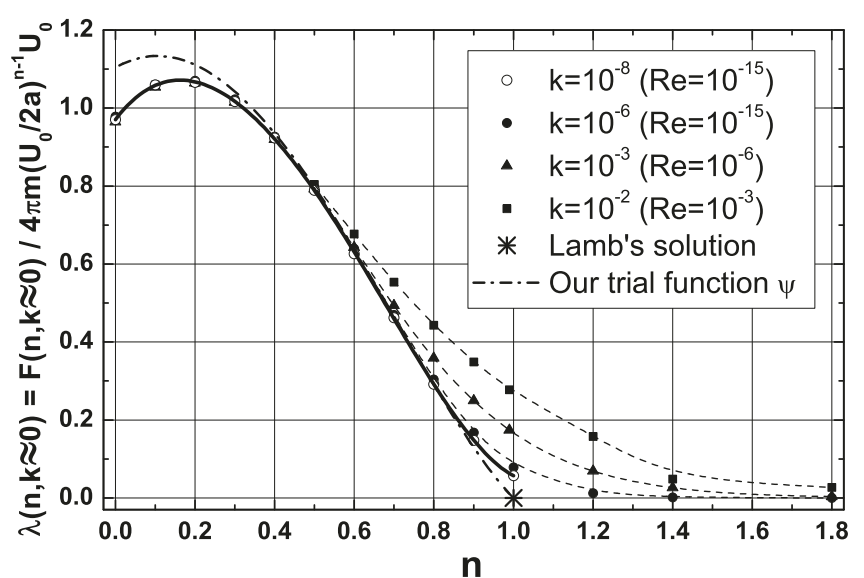

Fig. 5. Correction factor of the drag force undergone by a cylinder versus the index of fluidity $n$, showing the appearance of a Stokes-type solution due to the removal of the Stokes' paradox in shear thinning fluids and that due to the sensitivity to confinement for $n \geqslant 1$.

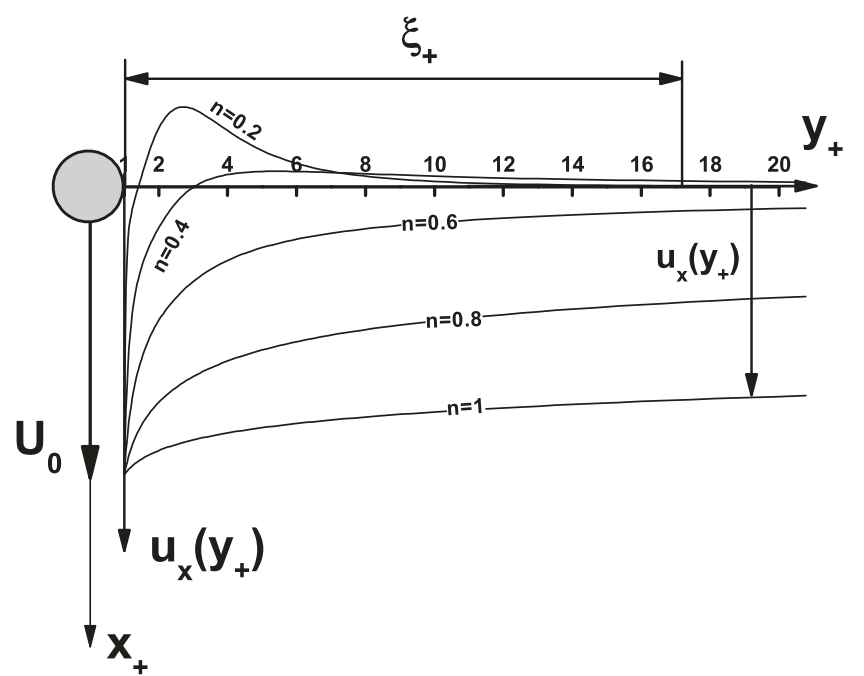

Fig. 6. Evolution of the velocity field and the screen length vs. the radial distance for different indexes $n$ in unbounded medium.

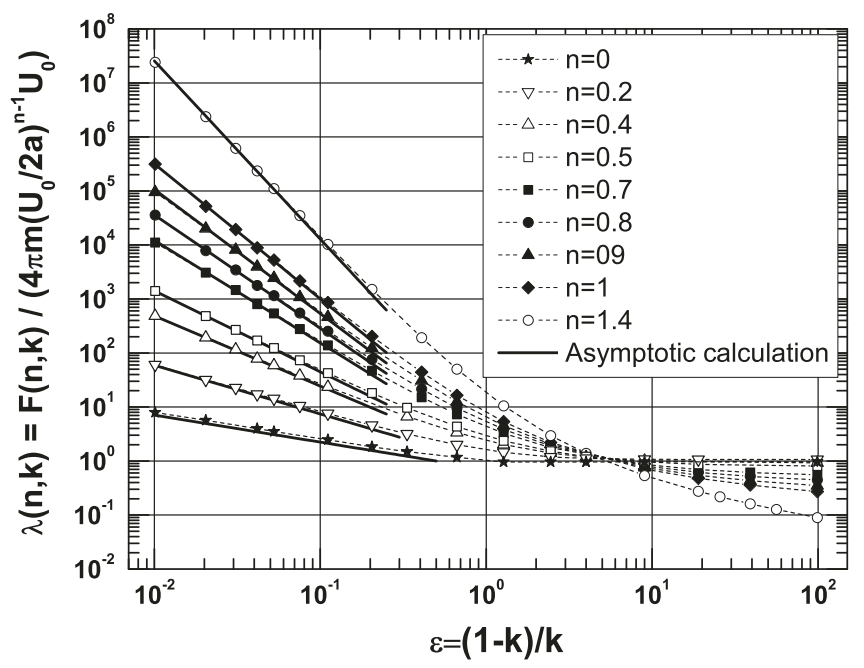

Fig. 7. Evolution of the drag correction factor $\lambda(n, k)$ undergone by a cylinder in a confined medium filled with a power-law fluid (numerical and asymptotical results). 

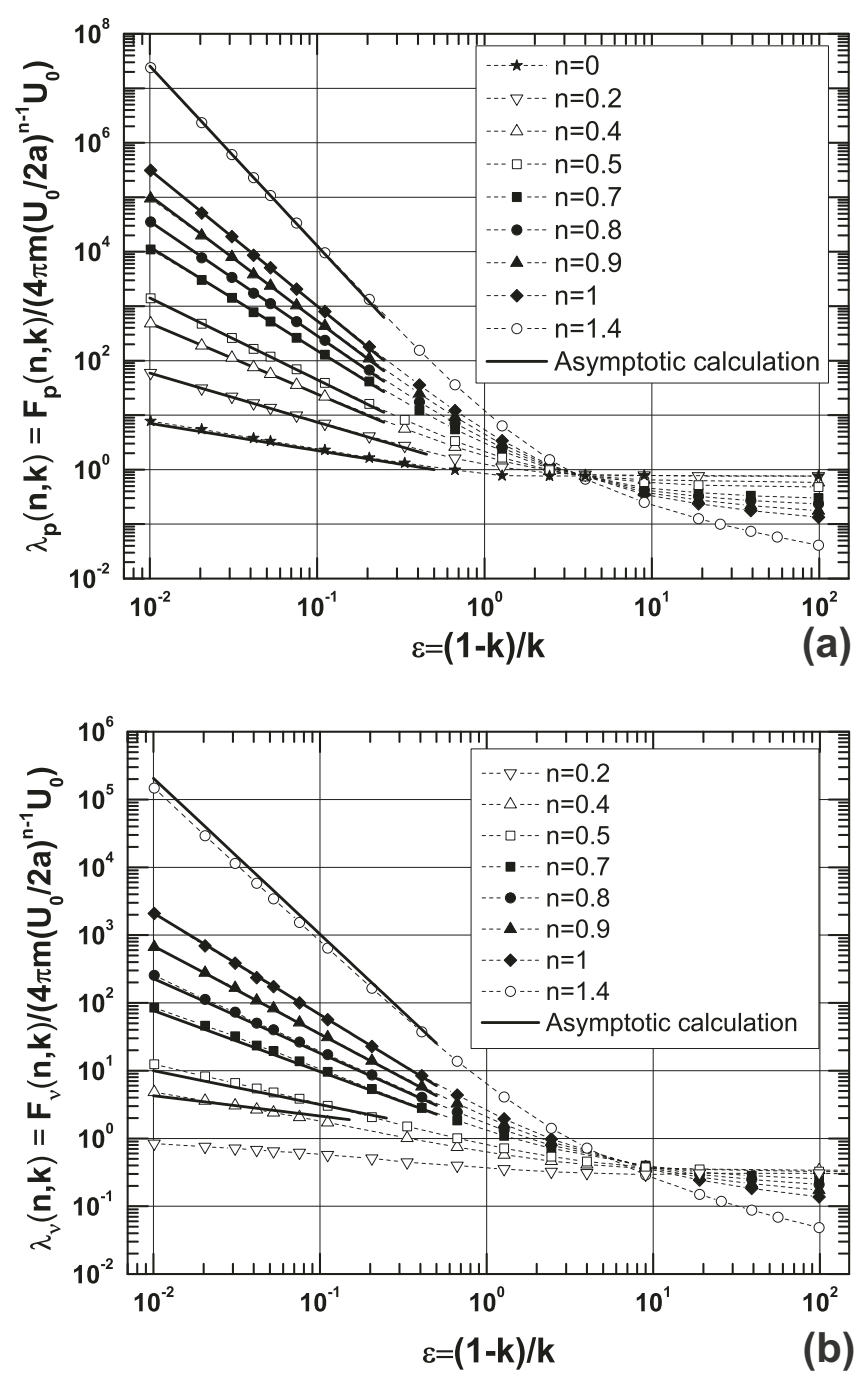

Fig. 8. Evolution of the contributions to the drag correction factor undergone by a cylinder in a confined medium filled with a power-law fluid (numerical and asymptotical results): (a) pressure contribution $\lambda_{p}(n, k)$, (b) viscous contribution $\lambda_{v}(n, k)$.

is the Rayleigh dissipation function for a power-law fluid. The minimization of $\lambda(n, k=0)$ was made by the use of "Mathematica". For $0.4 \leqslant n<1$, this method gives results which are in good agreement with the numerical ones and seems to give more accurate results for $0.9 \leqslant n \leqslant 1$, because they converge to the zero-value expected by Lamb's solution. Unfortunately, the results obtained by this method overestimate slightly the numerical results for $0 \leqslant n<0.4$. For this reason, we adopted the numerical procedure.

In conclusion, faced to all the results obtained, we propose a polynomial interpolation formula giving the values of $\lambda(n, k=0)$ for $0 \leqslant n \leqslant 1$ :

$$
\begin{aligned}
\lambda(n, k=0)= & 0.978+1.202 n-4.047 n^{2}+1.382 n^{3}+1.925 n^{4} \\
& -2.758 n^{5}+1.328 n^{6}
\end{aligned}
$$

Finally, in unbounded medium, the Fig. 5 shows that the drag force is zero for $n \geqslant 1$, where the Stokes' paradox takes place, due to the increase of the hydrodynamic screen length as shown in Fig. 6. This last figure also explains why, for $n<1$, the Stokes' paradox disappears as in a bounded medium, due to the decrease of the hydrodynamic screen length. Taking into account of all these results, and particularly the sensitivity to the inertia, we will give a study on the drag submitted by a cylinder in a confined situation, and we will calculate the relative velocity of the transportation of a free cylindrical particle in a power-law "Poiseuille flow". But before, let us give some results on the influence of the fluidity index on the sedimentation velocity of a fiber perpendicularly to its axis in two configurations.

\section{Sedimentation in power-law fluids}

\subsection{Cylindrical particles in a uniform bounded flow}

We are often concerned by the study of the hydrodynamic interactions between particles and walls as they arise during their sedimentation in Newtonian or non-Newtonian fluids, for example during the injection process of fiber-reinforced thermoplastics. To provide an estimation of the correction factor of this settling velocity, we give in Fig. 7 the correction factor for the drag undergone by a cylindrical particle in confined situation versus the normalized wall distance $\varepsilon=(1-k) / k=(b-a) / a$ for different fluidity indexes. Taking into account of the previous discussion concerning the sensitivity to the inertia, before any calculation, we verified that we are in the Stokes-type regime. To check the accuracy of the numerical results obtained in this problem, we proceed to an asymptotic approach in the lubrication regime to determine $\lambda(n, k)$ when the diameter of the cylinder becomes very close to the distance between both plane walls $(k \rightarrow 1)$. In this situation, all the dissipation is localized in the smallest gap, between the cylinder and the plane walls, of unknown limit (this effect has been confirmed by the numerical calculation of the vorticity in the transversal section of the cylinder). In this case, the drag forces can be estimated from the pressure and viscous forces induced by the backflow. In this Fig. 7, we can show the good agreement between the numerical calculations and the asymptotical ones, formula (8), even in dilatant fluids where the Stokes' paradox does not take place anymore due to the confinement $(n=1.4)$. We also remark that when $n$ decreases, the drag force is less and less sensitive to the wall hydrodynamic interactions, confirmed by the fact that the more the fluid is shear thinning, the more the screen length decreases (Fig. 6). Given these results, the study of the dynamics of fiber suspensions in non-Newtonian fluids has to take account of these interactions, especially when the fluid is dilatant. But, in pseudoplastic fluids, these interactions are all the more reduced so that $n$ is far smaller than one. In order to evaluate separately the contributions of the pressure and viscosity forces on the total drag, we compared in Fig. $8 \mathrm{a}$ and $\mathrm{b}$ the numerical and asymptotic calculations of $\lambda_{p}(n, k)$ and $\lambda_{v}(n, k)$ (formulas (8) and (9)) which are limited to the first term where $\epsilon_{0} / a$ is the smallest gap between the cylinder and the walls:

$$
\begin{aligned}
\lambda(n, k \rightarrow 1)= & \lambda_{p}(n, k \rightarrow 1)=\frac{2^{(4 n-1) / 2}}{\sqrt{\pi}}\left(\frac{1+2 n}{n}\right)^{n} \\
& \times \frac{\Gamma\left(2 n+\frac{1}{2}\right)}{\Gamma(2 n+1)}\left(\frac{\varepsilon_{0}}{a}\right)^{-(4 n+1) / 2}, \text { for } n \geqslant 0 \\
\lambda_{v}(n, k \rightarrow 1)= & \frac{2^{(6 n-5) / 2}}{\sqrt{\pi}}\left(\frac{1+2 n}{n}\right)^{n} \\
& \times \frac{\Gamma\left(2 n-\frac{1}{2}\right)}{\Gamma(2 n)}\left(\frac{\varepsilon_{0}}{a}\right)^{-(4 n-1) / 2}, \text { for } n>\frac{1}{4}
\end{aligned}
$$

Both Figs. $8 \mathrm{a}$ and $\mathrm{b}$ show that for high confinements $(k \approx 1)$, the pressure component dominates. In fact, $F_{p}$ dominates $F_{v}$ at any confinement in pseudoplastic fluids as in an unbounded medium. In the lubrication limit, we obtain a large pressure drop over the long length of the gap, much higher than the local viscous shear stresses. This pressure is exerted over the entire front and back surfaces of the cylinder, which is an area much greater than the one located in the small gap where the viscous shear stresses act. Thus the 

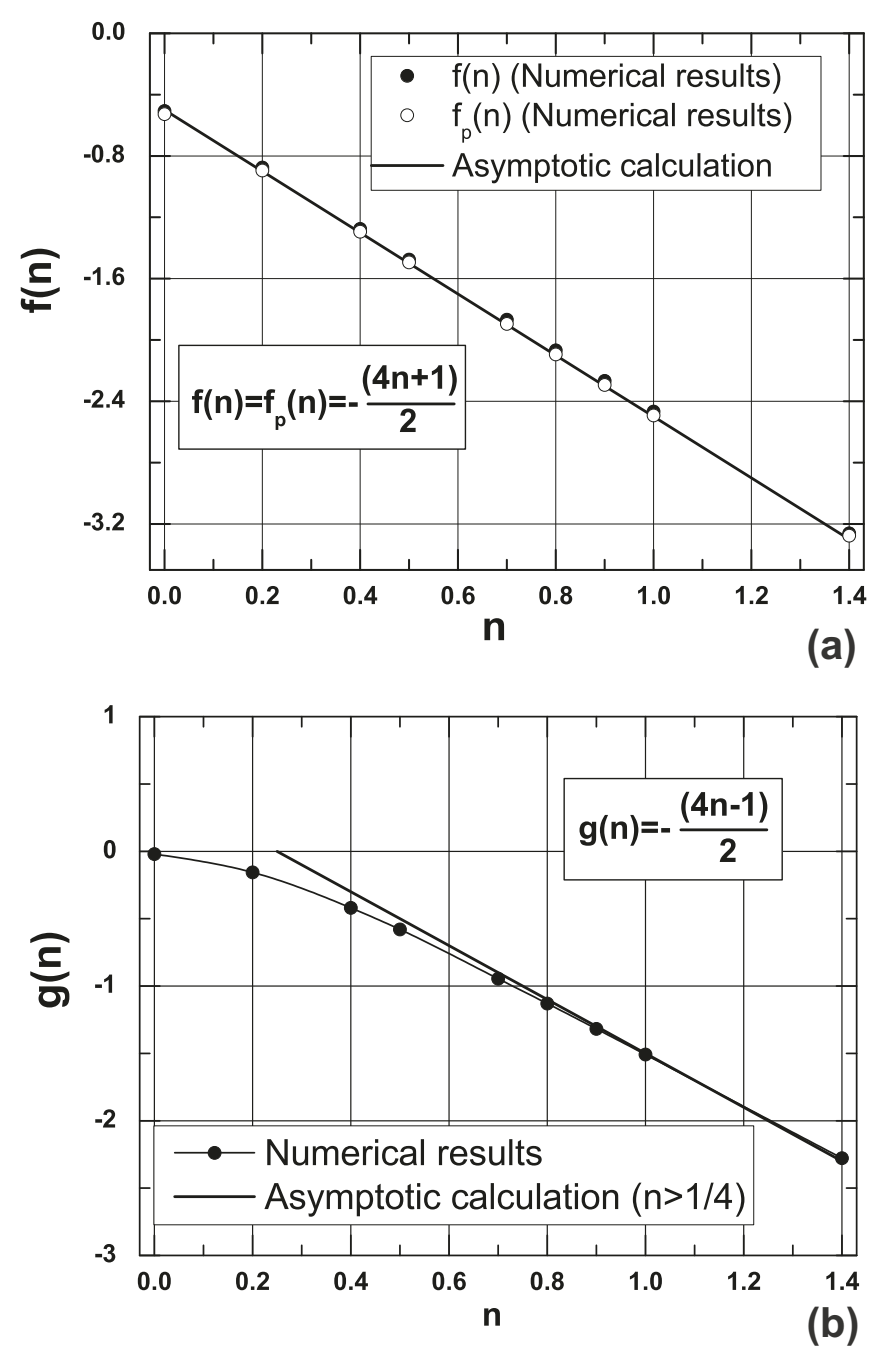

Fig. 9. Comparison of the numerical slopes $f(n)$ and $g(n)$ of the power-law behavior with the asymptotic one: (a) total and pressure drag force, (b) viscosity drag force.

pressure contribution to the drag force dominates the viscous contribution by orders of magnitude. In Fig. 8b, the limit of the asymptotic method to evaluate the viscous force for very low values of $n$ comes from the singular behavior of $\Gamma(x)$ function, which diverges at $x=0$ corresponding to $n=1 / 4$. Besides, the relative contribution of the pressure force over the viscosity one in the lubrication regime can be obtained by the ratio $R(n, k)=F_{p}(n, k \rightarrow 1) / F_{v}(n, k \rightarrow 1)$ through the following asymptotic formula:

$$
\begin{aligned}
R(n, k) & =\frac{F_{p}(n, k \rightarrow 1)}{F_{v}(n, k \rightarrow 1)}=\frac{\lambda_{p}(n, k)}{\lambda_{v}(n, k)} \\
& =\frac{1}{2^{n}}\left(\frac{4 n-1}{n}\right)\left(\frac{\varepsilon_{0}}{a}\right)^{-1}, \quad \text { for } n>\frac{1}{4}
\end{aligned}
$$

Finally, in Fig. 9a, we give a comparison of the exponent $f(n)$ of the power-law behavior of $\lambda_{p}(n, k)=\lambda(n, k) \propto\left(\varepsilon_{0} / a\right)^{f(n)}$ corresponding to the numerical results and the exponent expected theoretically by the asymptotical approach: $f(n)=-(4 n+1) / 2$. The good agreement confirms again the accuracy of both approaches. At the same time, we also compare in Fig. 9b the exponent $g(n)$ of the power-law variation $\lambda_{v}(n, k) \propto\left(\varepsilon_{0} / a\right)^{g(n)}$ to those predicted by the asymptotic calculation $g(n)=-(4 n-1) / 2$. In this curve, the slight deviation at low values of indexes of fluidity is due to the weakness of the estimation of the viscosity component by the asymptotic approach which probably needs the calculation of the higher order terms in
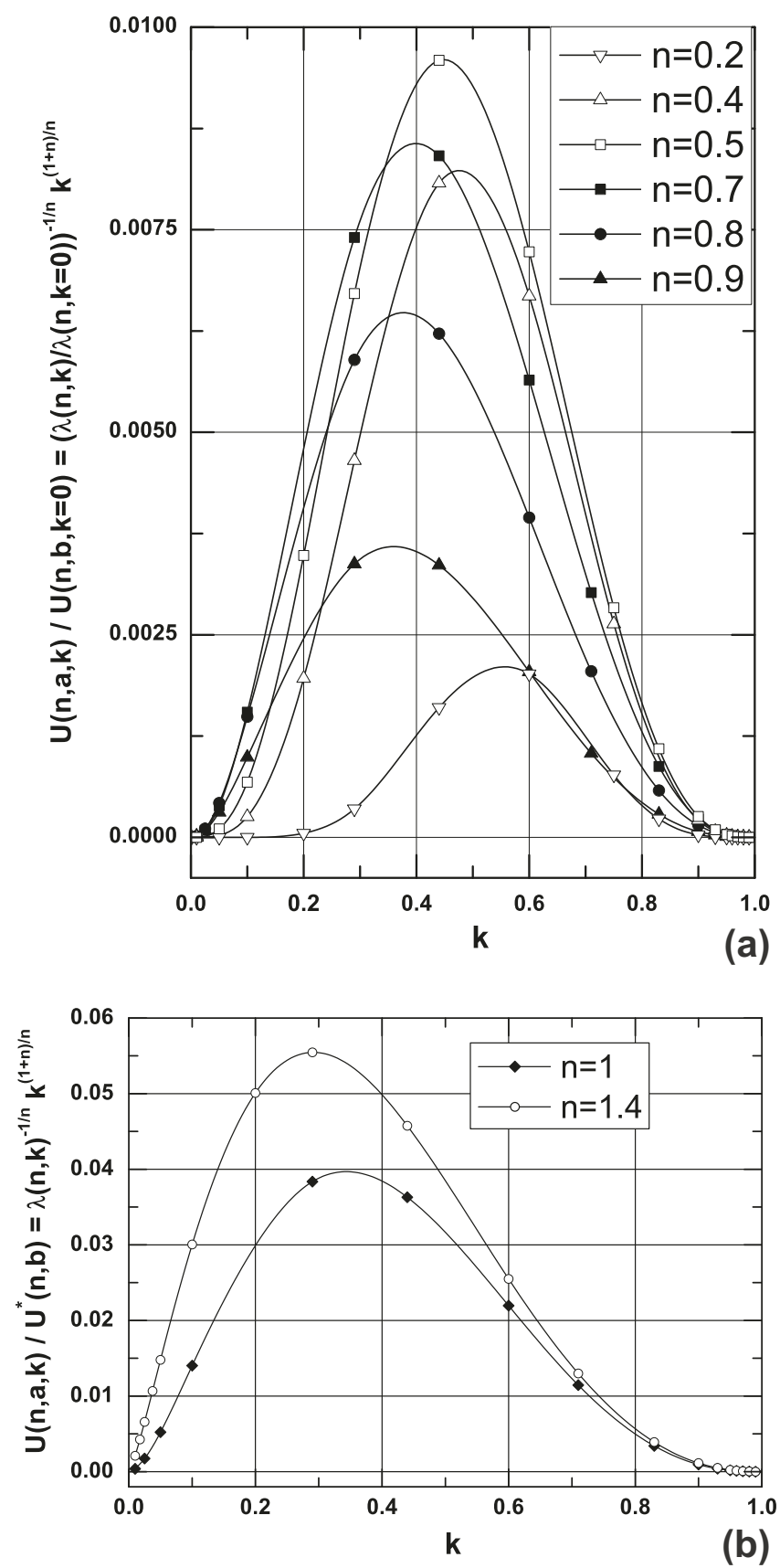

Fig. 10. Evolution of the normalized sedimentation velocity of a cylinder in a given container filled with a power-law fluid vs. its confinement factor $k$ : (a) when the Stokes' paradox does not take place, (b) when the Stokes' paradox appears in unbounded medium.

this range of $n$. In fact, for very shear-thinning fluids, those with $n<0.25$, the very high shear rates in the thin gap make the thinned viscosity negligibly small. In this condition, the leading contribution to the viscous stresses no longer comes from the small gap, but comes from the region away from the gap. Nevertheless, the numerical values remain accurate. In fact, for $n=0, g(n)$ must be equal to zero because $\lambda_{v}(n=0, k)$ is constant and equal to the value we obtained for the flow of a Bingham fluid at very high Bingham numbers as in an unbounded medium. At this step, we will give some applications of these results. The first one concerns the effect of the backflow on the settling velocity of a cylindrical particle in power-law fluids for a fixed distance between two walls. The second one concerns the sedimentation of a given cylindrical particle in a bounded medium for different confinements. 
4.2. Effect of the backflow on the particle settling velocity for different diameters in a given container

We consider a slit of rectangular cross section constituted by two parallel planes positioned at fixed distance $2 b$ and cylindrical particles of different diameters $2 a$ which are settling down perpendicularly to the gravity vector (Fig. 1). If this cylindrical particle is very long and highly confined, it has been established that the end effects are negligible [2,22]. For pseudoplastic fluids, for which the Stokes' type solution exists, we study the influence of the fluidity (n) and the backflow on the settling velocity. We plot in Fig. 10a the normalized sedimentation velocity by that achieved in an infinite medium $U(n, b, k=0)$ by a cylindrical particle of radius $b$, where the density difference is held constant:

$\frac{U(n, a, k)}{U(n, b, k=0)}=\left(\frac{\lambda(n, k=0)}{\lambda(n, k)}\right)^{1 / n} k^{(n+1) / n}$

with $\left.U(n, b, k=0)=\frac{2^{n-3}}{\lambda(n, k=0)} \frac{\Delta \rho g b^{n+1}}{m}\right)^{1 / n}$

In this curve, we show that, for a given distance between the parallel walls $2 b$, the sedimentation velocity of a cylindrical particle passes through a maximum for a critical diameter value $2 a_{c r}$. This striking effect is physically due to the competition between the increase of the particle weight as $a^{2}$ and the hydrodynamic friction generated by the backflow which is proportional to $a \lambda(n, a / b)$. At very low confinements $(k \approx 0)$, as the weight dominates, the asymptotic curve in the vicinity of $k=0$ is a power-law of the confinement: $\alpha(n, k) \propto k^{(n+1) / n}$. However, for high confinements $(k \approx 1)$, the asymptotic Eq. (8) gives us the asymptotic behavior:

$\frac{U(n, a, k)}{U(n, b, k=0)} \sim A(n)(1-k)^{(4 n+1) / 2 n}$

where

$A(n)=\left(\frac{n}{2 n+1}\right)\left[\lambda(n, k=0) \frac{\sqrt{\pi}}{2^{(4 n-1) / 2}} \frac{\Gamma(2 n+1)}{\Gamma(2 n+1 / 2)}\right]^{1 / n}$

In the pseudoplastic fluid, as the backflow depends on the fluidity index $n$, we observe that the more $n$ decreases from one to zero, the more the critical position of the maximum increases (Fig. 11a). Indeed, for lower values of $n$, the backflow needs only a very low gap between the particle and the wall because of the decrease of its hydrodynamic screen length (Fig. 6). The other noticeable effect is that the maximum of the settling velocity corresponding to this critical situation varies non-monotonically, passing through an optimum value for $n=0.55$. Besides, in the conditions where the Newtonian or dilatant fluid is confined, a solution of the Stokes-type exists but in an unbounded medium, the drag force is zero due to the fact that the Stokes' paradox takes place. In these conditions, the Fig. 10b shows the same behavior for the settling velocity as discussed above except the fact that the normalization is made by the use of a characteristic velocity $U^{*}(n, b)$ corresponding to a cylindrical particle of radius $b$ :

$\frac{U(n, a, k)}{U^{*}(n, b)}=\lambda(n, k)^{-1 / n} k^{(n+1) / n}$

where

$\left.U^{*}(n, b)=\frac{2^{n-3} \Delta \rho g b^{n+1}}{m}\right)^{1 / n}$

The consequence of these results is that during the sedimentation of polydisperse suspension, we expect fluctuations of settling velocity of each particle, all the more larger so that the confinement is important.
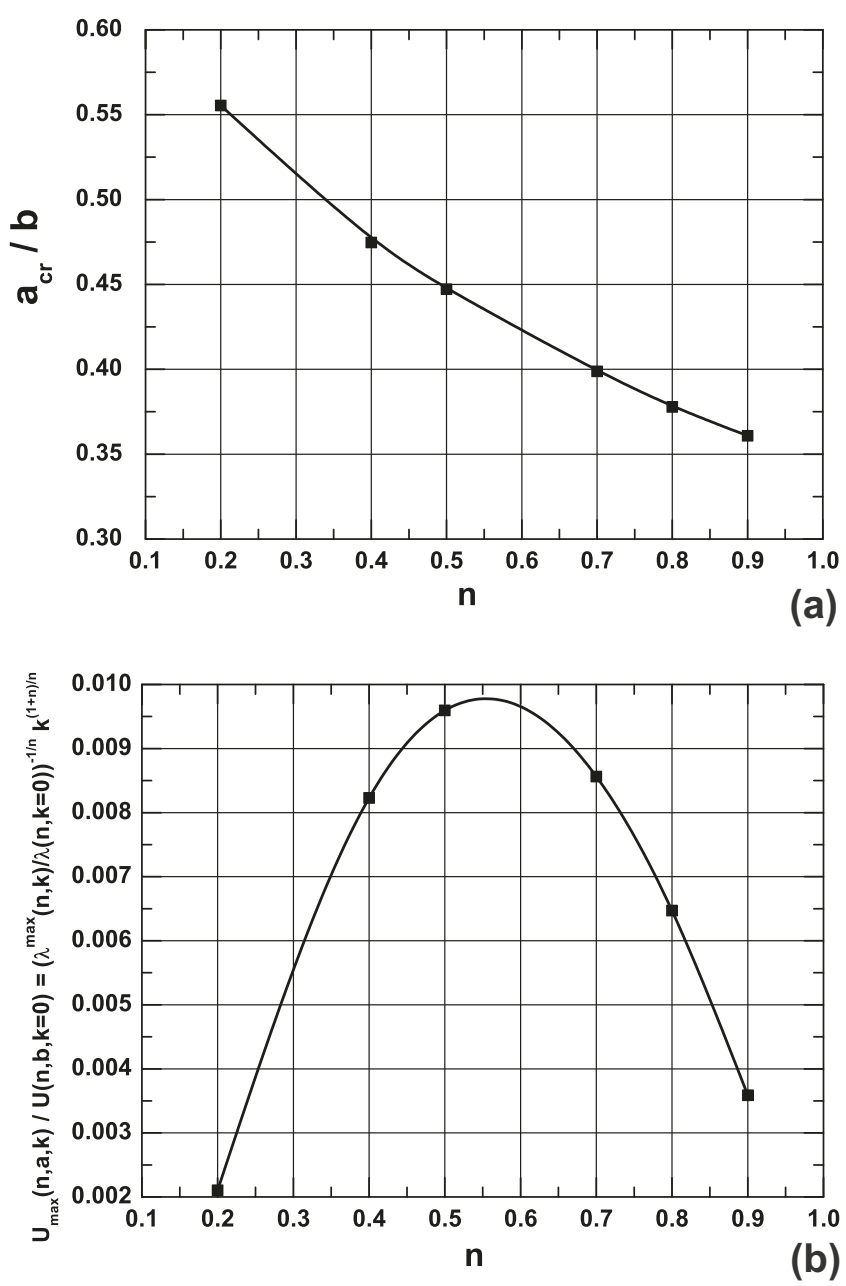

Fig. 11. (a) Evolution of the normalized critical radius of a cylinder sedimenting at its maximum velocity vs. the index $n$, (b) Evolution of the maximum normalized sedimentation velocity vs. the fluid index $n$.

Otherwise, to study the influence of the concentration on the settling velocity for a pseudoplastic fluid, we give in Fig. 12, for $0.4 \leqslant n<1$, the evolution of the effect of the confinement on the sedimentation velocity of a given cylindrical particle in a slit of rectangular cross section constituted by two parallel walls at different distances $2 b$. These results show that when the fluid becomes slightly shear thinning, the sedimentation velocity of fibers decreases strongly with the confinement. But this decrease is reduced when the fluid becomes more shear thinning $(n \ll 1)$.

\section{Effect of the confinement on the transportation velocity-shift}

In order to verify the validity of the assumption commonly used in some studies concerning the transportation of fibers (in injection processes for example), which consists in the free particle and the unperturbed fluid having the same velocity [23], we study the transportation velocity of a free non-buoyant cylindrical particle in the power-law fluid flow induced by a pressure gradient $\Delta P$ in a slit of aperture $2 b$. This hypothesis must be acceptable in dilute regime but as the concentrations used in industry are high, we expect that the hydrodynamic interactions coupled to the nonlinear rheological behavior induce a shift of this velocity which has not been studied yet to our knowledge. As the problem is fundamentally nonlinear, the determination of the real transportation velocity of the particle constitutes a nonlinear inverse problem which is difficult to solve. For these reasons, we first used a dynamic mesh 


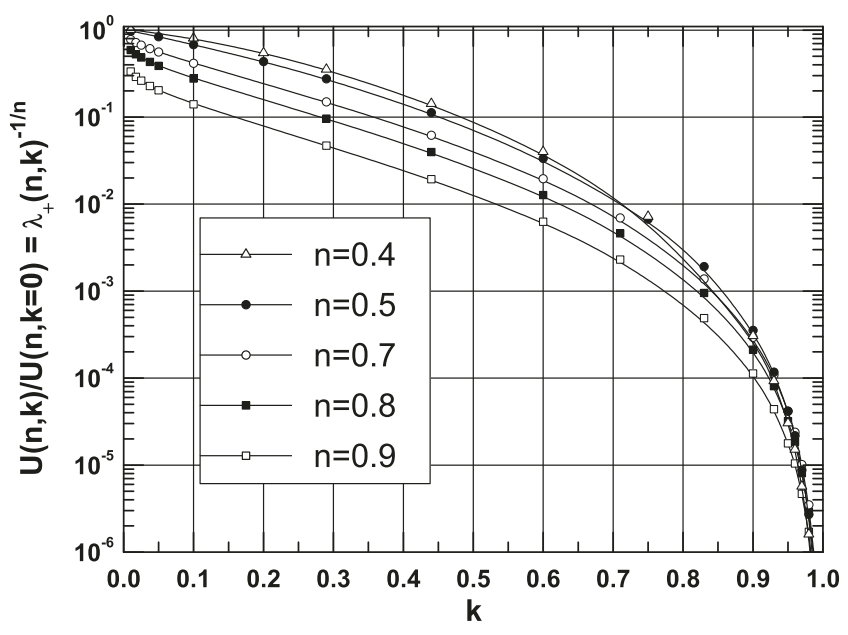

Fig. 12. Evolution of the settling velocity of a confined cylindrical particle, relative to the velocity achieved in unbounded medium.

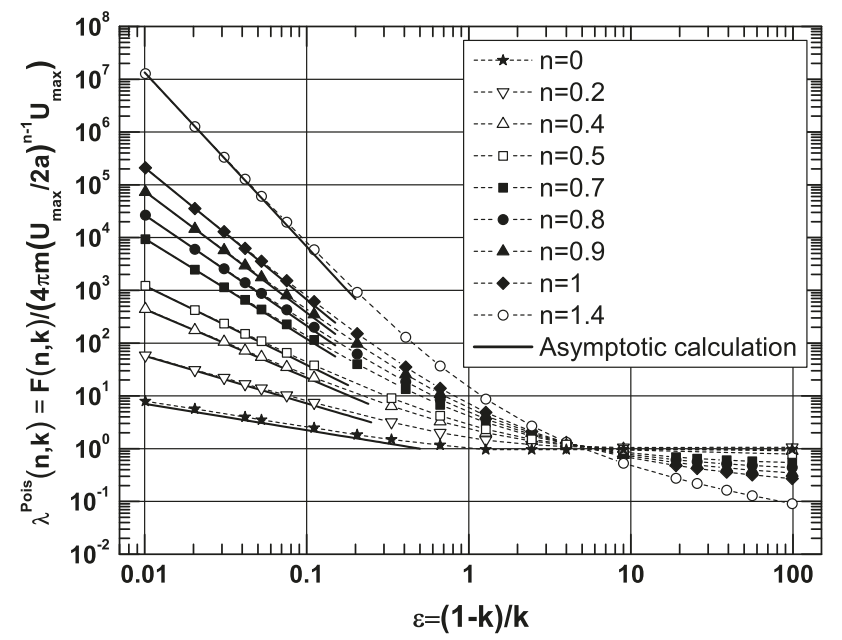

Fig. 13. Evolution of the drag correction factor $\lambda^{\text {Pois }}(n, k)$ undergone by a confined cylinder in the "Poiseuille type flow" of a power-law fluid (numerical and asymptotical results).

method described below. Even if the problem seems to be nonlinear, we tried to verify the validity of the use of the superposition of states.

\subsection{Dynamic mesh method}

To solve numerically this inverse problem, we used the dynamic mesh method available in the CFD FLUENT code. The numerical resolution used here is the same as described in Section 2.3. This dynamic mesh technique implies, for each time step, the rigid motion (which can be a translation or a rotation) of some boundaries relative to others. The mesh is then adjusted according to the new position of the moving boundaries. In this case, due to the symmetry and the invariance of the kinematics of the flow under translation, the dynamic mesh technique consists only in moving the mesh cells with the particle in order to avoid any deformation of the grid. The procedure used to solve this inverse problem consists in the determination of the translation velocity needed to be superposed to the unperturbed power-law fluid flow at which the drag force applied to the cylinder is reduced to zero corresponding to the free motion of the cylindrical particle. This critical velocity constitutes the actual transportation velocity shift.

\subsection{Cylindrical particle in a "Poiseuille" flow}

In the assumption of a very weak nonlinearity, the second method consists in the use of the superposition technique (as for Newtonian fluids [24]). The use of this method needs to determine the drag force undergone by a cylindrical particle placed in the symmetry plane in a power-law "Poiseuille cross flow". The fluid is driven by a pressure gradient $\Delta P / L$ corresponding to an imposed velocity profile, solution of the laminar flow of a power-law fluid in a slit, at the upstream and the downstream of the cylinder (avoiding the entrance effects):

$\boldsymbol{u}_{x}(y)=U_{\max }\left[1-\left(\frac{y}{b}\right)^{(1 / n)+1}\right] \boldsymbol{e}_{x}$

where $U_{\max }$ is the maximum value taken by the velocity in the power-law "Poiseuille flow". In these conditions, the forces exerted on a cylinder can be also written as:

$\boldsymbol{F}^{\text {Pois }}(n, k) / l=4 \pi m\left(\frac{U_{\max }}{2 a}\right)^{n-1} U_{\max } \lambda^{\text {Pois }}(n, k) \boldsymbol{e}_{x}$

In this section, to calculate the drag force, we used the same formulation and numerical method as described in Section 2, with an
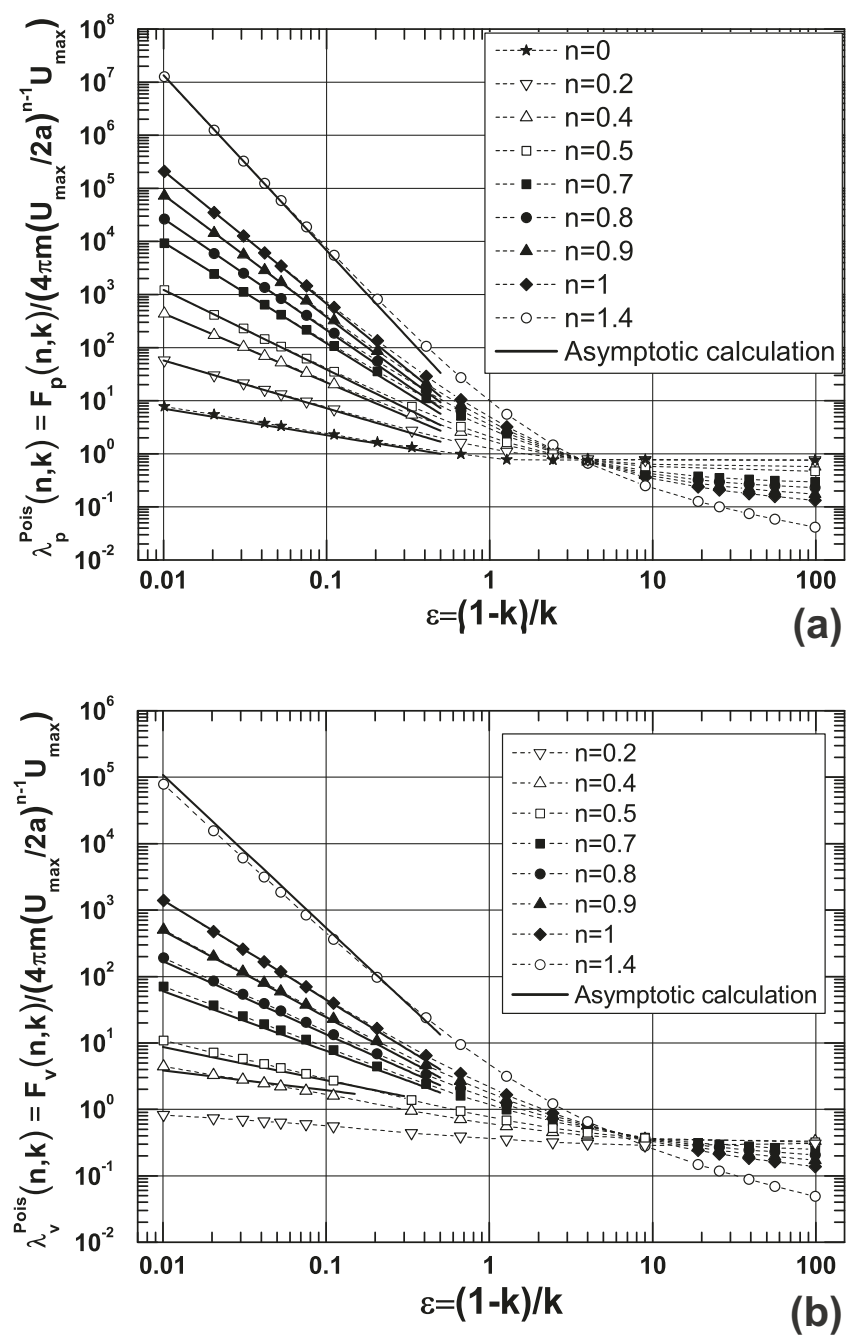

Fig. 14. Evolution of the contributions to the drag correction factor undergone by a confined cylinder in the "Poiseuille type flow" of a power-law fluid (numerical and asymptotical results): (a) pressure contribution $\lambda_{p}^{\text {Pois }}(n, k),(\mathrm{b})$ viscous contribution $\lambda_{v}^{\text {Pois }}(n, k)$. 


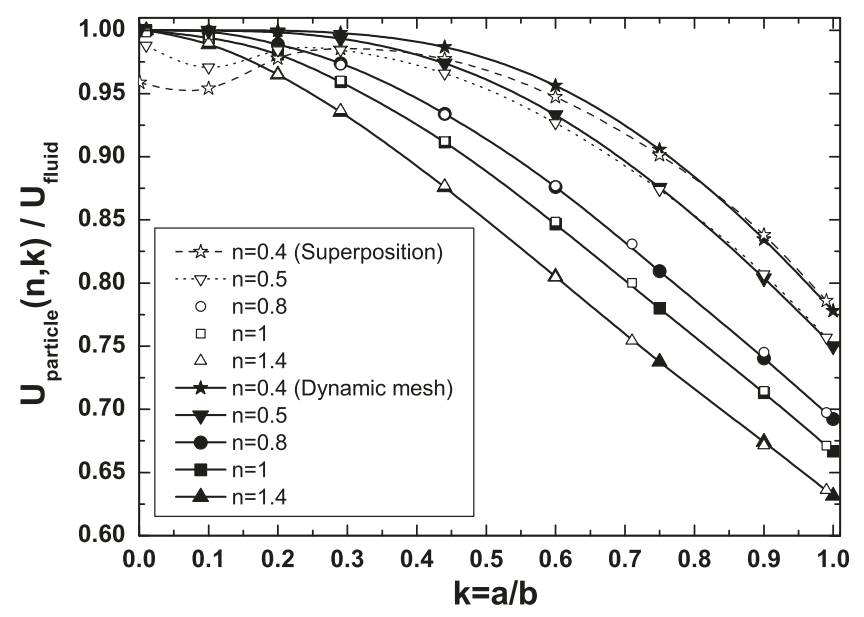

Fig. 15. Evolution of the velocity taken by a cylindrical particle transported in a "Poiseuille type flow" of a power-law fluid normalized by the unperturbed fluid velocity vs. its confinement.

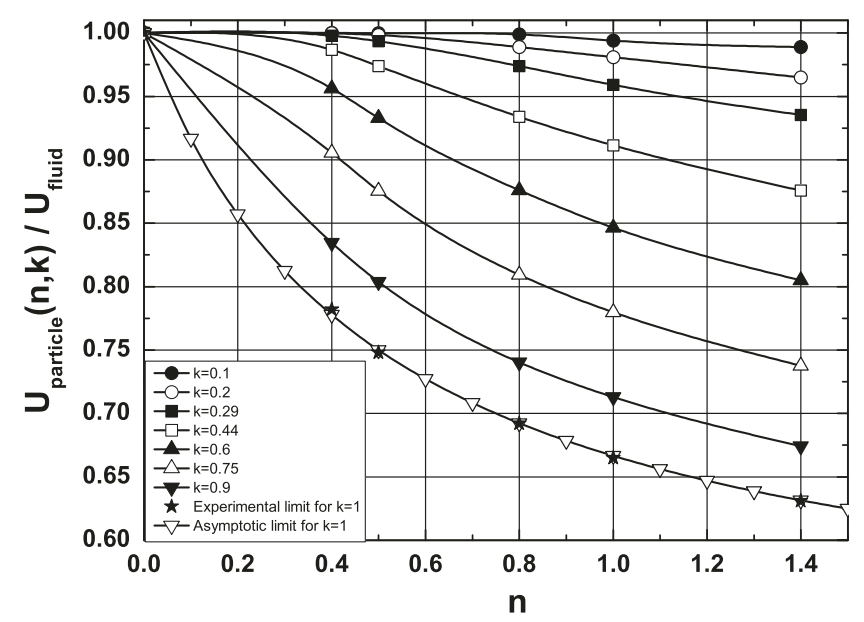

Fig. 16. Evolution of the velocity taken by a cylindrical particle transported in a "Poiseuille type flow" of a power-law fluid normalized by the unperturbed fluid velocity vs. its fluidity index $n$.

imposed velocity profile given in the formula (16) upstream and downstream far from the cylinder. In Fig. 13, we plotted the correction factor of the drag $\lambda^{\text {Pois }}(n, k)$. In the same way, the components of the pressure $\lambda_{p}^{\text {Pois }}(n, k)$ and the viscosity $\lambda_{v}^{\text {Pois }}(n, k)$ are given respectively in Fig. 14a and b. The successful comparison with the asymptotic formulas (18), (19) in this power-law "Poiseuille flow" confirms again the accuracy of the numerical results.

$$
\begin{aligned}
\lambda^{\text {Pois }}(n, k \rightarrow 1)= & \lambda_{p}^{\text {Pois }}(n, k \rightarrow 1)=\frac{2^{(4 n-1) / 2}}{\sqrt{\pi}}\left(\frac{n+1}{n}\right)^{n} \\
& \times \frac{\Gamma\left(2 n+\frac{1}{2}\right)}{\Gamma(2 n+1)}\left(\frac{\varepsilon_{0}}{a}\right)^{-(4 n+1) / 2}, \text { for } n \geqslant 0 \\
\lambda_{v}^{\text {Pois }}(n, k \rightarrow 1)= & \frac{2^{(6 n-5) / 2}\left(\frac{n+1}{n}\right)^{n}}{\sqrt{\pi}}\left(\frac{\Gamma\left(2 n-\frac{1}{2}\right)}{\Gamma(2 n)}\left(\frac{\varepsilon_{0}}{a}\right)^{-(4 n-1) / 2}, \text { for } n>\frac{1}{4}\right. \\
& \times \frac{\Gamma}{} \text {, }
\end{aligned}
$$

Nevertheless, the same remarks as in the uniform flow concerning the range of $n$ of validity of the viscosity component of the drag can be applied here. Otherwise, the same plot analysis concerning the slopes $f(n)$ and $g(n)$ given in the Fig. 9a and b is obtained for the power-law "Poiseuille flow".
Finally, if the superposition of states was permitted, then the flows corresponding respectively to the uniform and the powerlaw "Poiseuille flow" can be superposed to determine the translation velocity of a free particle at which the drag force is zero.

$$
\begin{aligned}
F_{x} / l= & -4 \pi m\left(\frac{U_{\text {particle }}}{2 a}\right)^{n-1} U_{\text {particle }} \lambda(n, k) \\
& +4 \pi m\left(\frac{U_{\max }}{2 a}\right)^{n-1} U_{\max } \lambda^{\text {Pois }}(n, k)=0
\end{aligned}
$$

Then, the transportation velocity of the free particle normalized by the velocity of the unperturbed fluid at the symmetry plane of the slit $U_{\text {fluid }}=U_{\max }$ in the power-law "Poiseuille flow" is plotted against the confinement factor in Fig. 15. For all fluidity indexes $n$, the velocity shift of the particle increases with the confinement (i.e. the concentration) due to the hydrodynamic interactions with the plane walls. Otherwise, it is surprising that the results obtained by the superposition method are in good agreement with those obtained by the direct dynamic mesh technique for $0.7 \leqslant n \leqslant 1.4$. This fact confirms the validity of the hypothesis of the weak nonlinearity of the power-law fluid problem. However, for $n=0.5$ and $n=0.4$ (i.e. the low values of $n$ ), the dynamic mesh seems to give good results but the superposition method seems not to be usable anymore with the exception of $k \approx 1$. In fact, in the Fig. 16, where we give this shift velocity versus the index of fluidity for different confinements, we can show that the accuracy of the calculation can be proved by the good value obtained in the limit of $k \approx 0$ (very dilute regime). We show also that in the limit of $k \approx 1$, the particle moves at the same mean fluid velocity in the slit in which:

$\frac{U_{\text {particle }}}{U_{\text {fluid }}}=\frac{n+1}{2 n+1}$

The results in Fig. 15 prove finally that the shift of the particle velocity relative to that of the fluid is more important than the Newtonian fluid in dilatant fluids but it is reduced for high concentrations in pseudoplastic fluids when the fluid becomes more and more shear thinning. The consequence of these last results is that during the injection of the fibers in polymers, the error committed using Tucker's hypothesis is reduced for very low values of $n$. However, for dilatant fluids, this error is amplified. These remarks concerning the shift of velocity can be applied to the transportation of any particle in non-Newtonian fluids having a shear thinning or shear thickening behavior.

\section{Conclusion}

In this study, we provided a numerical solution to the Stokestype problem concerning the drag submitted by a cylinder in a power-law fluid. Indeed, once the sensitivity to the inertia and the confinement has been clarified, we showed that in a pseudoplastic unbounded medium, the Stokes-type solution exists due to the fact that the Stokes' paradox does not take place anymore. Then, we give numerical results for this problem, for which all the data are not available yet. At this occasion, we give a physical explanation of this surprising effect which is due to the "virtual hydrodynamic confinement" of the flow induced by the reduction of the hydrodynamic screen length when $n<1$. Besides, in dilatant fluids, we show that the drag force in unbounded medium is zero due to the Stokes' paradox which still takes place for $n \geqslant 1$. This is due to the increase of the screen length for Newtonian and dilatant fluids. When the problem is confined geometrically, we showed that the drag force is not anymore zero due to the existence of the Stokes-type solution as established in the Newtonian case [2]. Otherwise, once the solution for the unbounded medium is established, we give a solution for the confined medium 
numerically and asymptotically. The successful comparison, which confirms the validity of the calculation, leads us to give a solution of the settling of a fiber perpendicularly to the gravity vector, and particularly in a given slit. Using the dynamic mesh technique, we solved the inverse nonlinear problem consisting on the determination of the shift velocity of a non-buoyant cylindrical particle conveyed by a power-law "Poiseuille flow", induced by the hydrodynamic interactions. These results show that, the more $n$ decreases, the more the shift velocity is reduced in comparison to the Newtonian case. To verify the strength of the nonlinearity of this problem, we compare the numerical results with those obtained by the technique of superposition which is valid only for very weak nonlinearities. This assumption seems to be acceptable only for large values of $n$. The consequence of this last result on the transportation of particles is that during the injection process of fibers, the attenuation of the relative velocity with that of the nonperturbed fluid particle for pseudoplastic fluids reduces the effect of hydrodynamic interactions.

\section{References}

[1] M. Sepehr, G. Ausias, J.P. Carreau, Rheological properties of short fibers filled propylene in transient shear flow, J. Non-Newtonian Fluid Mech. 123 (2004) 19-32.

[2] S. Champmartin, A. Ambari, Kinematics of a symmetrically confined cylindrical particle in a stokes-type regime, Phys. Fluids 19 (2007) 073303-1-073303-11.

[3] M.K. Lyon, D.W. Mead, R.E. Elliot, L.G. Leal, Structure formation in moderately concentrated viscoelastic suspensions in simple shear flow, J. Rheol. 45 (4) (2001) 881-890.

[4] R. Scirocco, J. Vermant, J. Mewis, Effect of the viscoelasticity of the suspending fluid on structure formation in suspensions, J. Non-Newtonian Fluid Mech. 117 (2004) 183-192.

[5] G.G. Stokes, On the effect of the internal friction of fluids on the motion of pendulums, Trans. Camb. Philos. Soc. 9 (II) (1851) 8-106
[6] C.W. Oseen, Uber die stokessche formel und ber eine verwandte aufgabe in der hydrodynamic, Ark. Mat. Astron. Fys. 6 (1) (1910) 29.

[7] H. Lamb, On the uniform motion of a sphere through a viscous fluid, Philos. Mag. 21 (121) (1911) 112-121.

[8] G.K. Batchelor, Slender-body theory for particles of arbitrary cross section in stokes flow, J. Fluid Mech. 44 (3) (1970) 419-440.

[9] R.I. Tanner, Stokes paradox for power-law flow around a cylinder, J. NonNewtonian Fluid Mech. 50 (1993) 217-224.

[10] E. Marušić-Paloka, On the stokes paradox for power-law fluids, Z. Angew. Math. Mech. 81 (1) (2001) 31-36.

[11] A. Ben Richou, A. Ambari, J.K. Naciri, Drag force on a circular cylinder midway between two parallel plates at very low reynolds numbers - part 1: Poiseuille flow (numerical), Chem. Eng. Sci. 59 (2004) 3215-3222.

[12] R. Peyret, T.D. Taylor, Computational Methods for fluid flow, Springer-Verlag, 1985

[13] L. Quartappelle, Vorticity conditioning in the computation of two dimensional viscous flows, J. Comput. Phys. 40 (1981) 453-477.

[14] D.W. Peaceman, H.H. Rachford, The numerical solution of parabolic and elliptic differential equation, J. Soc. Ind. Appl. Math. 3 (1955) 28-44.

[15] J. Douglas, J.E. Gunn, A general formulation of alternating direction method, Num. Mathematik 6 (1964) 428-453.

[16] S.P. Frankel, Convergence rates of iterative treatments of partial differential equations, Math. Tables Aids Comput. 4 (1950) 65-75.

[17] J.M. Ferreira, R.P. Chhabra, Analytical study of drag and mass transfer in creeping power law flow across tube banks, Ind. Eng. Chem. Res. 43 (2004) 3439-3450.

[18] M.J. Whitney, G.J. Rodin, Force-velocity relationships for rigid bodies translating through unbounded shear-thinning power-law fluids, Int. J. NonLinear Mech. 36 (2001) 947-953.

[19] P. Sivakumar, R.P. Bharti, R.P. Chhabra, Steady flow of power-law fluids across an unconfined elliptical cylinder, Chem. Eng. Sci. 62 (2007) 1682-1702.

[20] Y. Tomita, On the fundamental formula of non-newtonian flow, Bull. Japan. Soc. Mech. Eng. 2 (1959) 469-474.

[21] M.L. Wasserman, J.C. Slattery, Upper and lower bounds on the drag coefficient of a sphere in a power-law fluid, AIChE J. 10 (1964) 383-388.

[22] B. semin, J.P. Hulin, H. Auradou, Influence of flow confinement on the drag force on a static cylinder, Phys. Fluids 21 (2009) 103604.

[23] C.L. Tucker, Flow regimes for fiber suspensions in a narrow gap, J. NonNewtonian Fluid Mech. 39 (1991) 239-268.

[24] J. Happel, H. Brenner, Low Reynolds Number Hydrodynamics: with special applications to particulate media, Springer, 1983. 